\title{
Preparation of $\mathrm{Gd}_{2} \mathrm{O}_{3}: \mathrm{Er}, \mathrm{Yb}$ Nanoparticles by Laser Ablation in Liquid and Their Optical Properties for Biomedical Application
}

\author{
Yuri Tei ${ }^{* 1}$, Haohao Wang ${ }^{2}$, Yoshitaka Kitamoto ${ }^{1}$, Masahiko Hara ${ }^{1}$, and Hiroyuki Wada ${ }^{1}$ \\ ${ }^{1}$ School of Materials and Chemical Technology, Tokyo Institute of Technology, Japan \\ ${ }^{2}$ Department of Physics, Nanjing Agricultural University, China \\ ${ }^{*}$ Corresponding author's e-mail: tei.y.aa@m.titech.ac.jp
}

\begin{abstract}
$\mathrm{Gd}_{2} \mathrm{O}_{3}$ :Er,Yb upconversion nanoparticles were obtained by laser ablation in liquid, and their optical properties were investigated. These nanoparticles are suitable for photodynamic therapy which is a cancer treatment approach. Since the nanoparticles are for medical use, purity is required. Thus, laser ablation in liquid was selected as a suitable production method because it produces no impurities. Nanoparticles were prepared by Nd:YAG (532-nm) laser-irradiation to $\mathrm{Gd}_{2} \mathrm{O}_{3}$ :Er, Yb target in water. The nanoparticles' compositions were analyzed using X-ray diffraction, which showed that the nanoparticles and target raw material have the same peak. The primary particle size was measured using scanning electron microscopy, which revealed the coarse $(>100 \mathrm{~nm})$ and fine $(<100 \mathrm{~nm})$ nanoparticles. The secondary particle size was investigated by dynamic light-scattering, which indicated the aggregation of fine nanoparticles. Upconversion luminescence and photon number were measured using a photoluminescence spectrometer with 980-nm laser excitation. Visible light was obtained due to upconversion. The photoluminescence intensity increased until the middle fluence and slightly decreased at the high fluence. Besides, the photon number was increased from 2.3 to 3.2. It is considered that nonradiative deactivation of fine nanoparticles affected the emission characteristics and photon number.
\end{abstract}

DOI: $10.2961 /$ jlmn.2021.02.2007

Keywords: nanoparticle, upconversion, $\mathrm{Gd}_{2} \mathrm{O}_{3}$, laser ablation, nanosecond laser, near-infrared light, photodynamic therapy

\section{Introduction}

Laser processing is one of promising techniques in various research fields. Laser surface structuring, which is one of attractive laser processing, is applied to a wide range of technologies such as semiconductors, integrated circuits, solar cells, LEDs, and digital sensors [1]. As a recent new technology using laser, N. Livakas et al. proposed technique for fabrication of large area of multi-directional laser-induced periodic surface structures (LSFL) [2]. Laser ablation is another laser processing technology, and it is a method for producing thin films and nanoparticles that has been growing rapidly in the last 10 years. Laser ablation in liquid (LAL) is a method that can obtain colloids with high productivity [3-7]. LAL is of great interest in the fields of catalysis, biomedicine, cancer therapy, 3D printing, analytical chemistry and so on.

The nanoparticles (NPs) can be physically formed from raw materials by LAL. The method is advantageous because it is simple and does not produce impurities. LAL is suitable for producing NPs for medical materials, which require high safety [8-9]. L. Chen et al. reported silicon nanoparticles with different doping concentrations by LAL and their optical properties [10]. One of the problems to be solved in LAL is the elucidation of the mechanism of nanoparticle formation, which has been studied by S. Dittich et al. on cavitation bubbles [3].

Upconversion is a phenomenon whereby short wavelength light (high energy) can be converted from a long wavelength light (low energy) [11-15]. Regarding the ener- gy diagram, for a two-photon absorption process, the upconversion material absorbs two photons from irradiated light and is excited to the second excited state. Afterward, it emits fluorescence, which is the upconverted light, while returning to the ground state. Applications of upconversion NPs include solar cells, photocatalysts, cancer treatments, and bioimaging [11-12, 16-20].

Unlike other cancer treatment methods, photodynamic therapy (PDT) has fewer side effect than typical treatment, such as chemotherapy or radiotherapy. At one location, repeated PDT can be used, which is rather difficult with radiotherapy. Furthermore, PDT requires a less invasive procedure than surgical treatments and produces little or no scars [21-23].

In conventional PDT, a photosensitizer is used to destroy the cancer cells. The photosensitizer is activated by irradiated visible light and generates reactive oxygen species (ROS). The generated ROS kill the cancer cells to treat cancer. However, visible light $(400-800 \mathrm{~nm})$ is absorbed by the human body because it is not within the biological window (600-1000 nm) [24]. Therefore, conventional PDT is only used for cancer problems (skin and lungs) located in shallow areas (several nm from the surface of the skin [25]). However, if upconversion NPs can be used in cancer cells and near-infrared light ( $800-2500 \mathrm{~nm})$ is unabsorbed by the human body, visible light can be obtained inside the cancer cells, and PDT can be performed. If PDT with upconversion NPs is realized, it is expected that deep (several cen- 
timeters from the surface of the skin [26]) and largevolume cancer cells can be treated.

Required conditions for upconversion NPs for PDT are the excitation wavelength within 800-2500 nm (nearinfrared light) to penetrate the body and emission wavelength within 400-800 nm (visible light) to activate photosensitizers.

As upconversion materials, rare-earth doped NPs can be excited by near-infrared light, which is within the biological window, and emit visible light. As a host material, Gadolinium oxide $\left(\mathrm{Gd}_{2} \mathrm{O}_{3}\right)$ was selected for its high thermal and chemical stability, and low phonon energy (small lattice vibration, which does not disturb excitation or emission process of lanthanide) [27-28]. As a doping material, $\mathrm{Yb}^{3+}$ and $\mathrm{Er}^{3+}$ were selected because $\mathrm{Yb}^{3+}$ can be excited with near infrared light and $\mathrm{Er}^{3+}$ can emit visible light (strong emission in red: $660 \mathrm{~nm}$, green: $540 \mathrm{~nm}$ and 565 $\mathrm{nm}$, blue: $410 \mathrm{~nm}$ ) [29-30]. $\mathrm{Gd}_{2} \mathrm{O}_{3}$, which is also used as an MRI contrast agent, has been reported to have low cytotoxicity [31]. Furthermore, the results of a previous study by Ikehata et al. on the cell viability of $\mathrm{Y}_{2} \mathrm{O}_{3}: \mathrm{Er}, \mathrm{Yb}$ NPs was found to be high when the nanoparticle concentration was less than $1 \mathrm{mg} / \mathrm{mL}$ [32]. Therefore, it is considered that rare earth elements are not toxic to the ecology.

Regarding the particle size, $10-200 \mathrm{~nm}$ is required for selective accumulation in cancer cells from the aspect of circulation in the body and enhanced permeation and retention effect (EPR effect). NPs are usually injected into blood vessels and circulate in the body. While circulating, NPs of sizes $<10 \mathrm{~nm}$ (excreted by the kidney) [33] and $>500 \mathrm{~nm}$ (excreted by the liver) [34] are excreted from the body. Therefore, 10-500-nm sized NPs can circulate in the body. Moreover, since blood vessels' gaps of 200-nm width exist around cancer tissues, if NPs smaller than $200 \mathrm{~nm}$ can selectively accumulate in cancer cells (EPR effect) [15, 3435].

This study aims to prepare 10-200-nm sized $\mathrm{Gd}_{2} \mathrm{O}_{3}: \mathrm{Er}, \mathrm{Yb}$ NPs by LAL and investigate their optical properties.

\section{Methods and characterization}

\subsection{Preparation of $\mathrm{Gd}_{2} \mathrm{O}_{3}: \mathrm{Er}, \mathrm{Yb}$ pellet}

The reagents used for the experiment were $\mathrm{Gd}\left(\mathrm{NO}_{3}\right)_{3} \cdot$ $6 \mathrm{H}_{2} \mathrm{O}$ (Kanto Chemical Co., Ltd.), $\mathrm{Er}\left(\mathrm{NO}_{3}\right)_{3} \cdot 5 \mathrm{H}_{2} \mathrm{O}$ (Sanwa Chemical Industries), $\mathrm{Yb}\left(\mathrm{NO}_{3}\right)_{3} \cdot 5 \mathrm{H}_{2} \mathrm{O}$ (Wako Pure Chemical Industries, Ltd.), and $\mathrm{NH}_{4} \mathrm{OH}$ (28\%) (Kanto Chemical Co., Inc.).

$$
\mathrm{Gd}\left(\mathrm{NO}_{3}\right)_{3} \cdot 6 \mathrm{H}_{2} \mathrm{O} \text { (89.0-mol\%), } \mathrm{Er}\left(\mathrm{NO}_{3}\right)_{3} \cdot 5 \mathrm{H}_{2} \mathrm{O}
$$
(1.00-mol\%), and $\mathrm{Yb}\left(\mathrm{NO}_{3}\right)_{3} \cdot 5 \mathrm{H}_{2} \mathrm{O}$ (10.0-mol\%) were dissolved in deionized water $(50-\mathrm{mL})$ and sonicated for 30 min for complete dissolution. Aqueous ammonia solution (11-mL) was added dropwise with stirring. The reaction was performed for $2 \mathrm{~h}$ under continuous stirring, and the resulting solution was aged for $24 \mathrm{~h}$. Next, the sediment was washed with water and dried at $60^{\circ} \mathrm{C}$ for $12 \mathrm{~h}$. Furthermore, the precursor was fired at $900^{\circ} \mathrm{C}$ for $2 \mathrm{~h}$ in an electric furnace. The powder was pressed at room temperature, under $140 \mathrm{MPa}$ of pressure, for 5 min to obtain a pellet. Afterward, the pellet was sintered at $1200^{\circ} \mathrm{C}$ for $2 \mathrm{~h}$ in an electric furnace.

\subsection{Fabrication of $\mathrm{Gd}_{2} \mathrm{O}_{3}$ : Er, Yb NPs}

The pellet was added to deionized water $(6-\mathrm{mL})$, and the resulting mixture was irradiated by Nd: YAG laser (second harmonic generation (SHG), 532-nm wavelength, $10-\mathrm{Hz}$ frequency, and 13-ns pulse width), a focused pulsed laser beam. The circle formed by laser irradiation was $1.7 \mathrm{~mm}$ long and $1.7 \mathrm{~mm}$ wide. The laser intensity (fluence) was changed to $2.4,4.7,6.4$, and $8.9 \mathrm{~J} / \mathrm{m}^{2}$. The irradiation time at each fluence was $30 \mathrm{~min}$.

\subsection{Characterization}

The compositions of the raw materials and NPs (6.4 $\mathrm{J} / \mathrm{cm}^{2}$ ) were analyzed using X-ray diffraction (XRD,

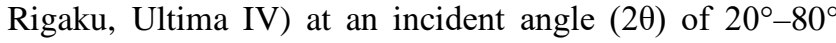
with $\mathrm{Cu}$ tube radiation.

The primary particle size was measured using scanning electron microscopy (SEM, Hitachi High technologies, S4800). The observation was performed while filling the SEM equipment with liquid nitrogen for a clear SEM image. Measurement sample of a raw material pellet was prepared by partially crushing the pellets and placing the fragments on a carbon tape. The measurement samples of NPs were made using a copper grid coated with a carbon support film and dropping a small amount of the supernatant of the nanoparticle suspension.

The secondary particle size of the prepared NPs was measured using a dynamic light-scattering system (DLS, Sysmex Co, Zetasizer Nano). The measurement samples were prepared by collecting a 1-mL quantity from the supernatant of the nanoparticle suspension at each fluence.

The fluorescence intensity of the NPs was measured using a photoluminescence (PL) spectrometer (PL, HITACHI, F-7000). The photon number was measured from the change in fluorescence intensity when the current value was changed. A laser diode (THORLABS, L980P300J), a laser mount (HORLABS, TCLDM9), and a temperaturecurrent controller (THORLABS, LTC100 B) were used as the light source for the excitation light. The measurement conditions were 980-nm excitation wavelength, 700-V photomultiplier voltage, $1.0-\mathrm{nm}$ slit, and 400-800-nm monitoring wavelength. The measurement samples were prepared by collecting a 1-mL quantity from the supernatant of the nanoparticle suspension prepared at each fluence. A quartz cell was used for the measurement.

\section{Results and discussion}

\subsection{Crystal structure}

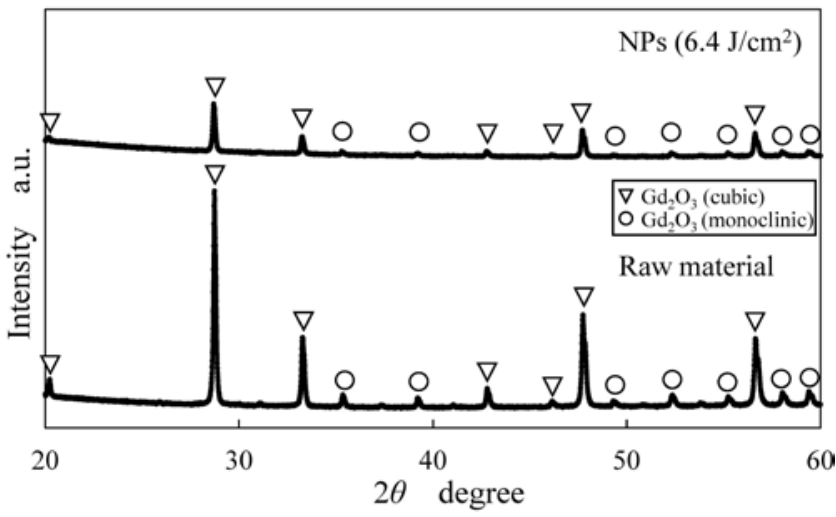

Fig. 1 XRD patterns of (a) target and (b) prepared NPs. 
Figure 1 shows the results of the compositional analysis by XRD. The upper and lower rows represent the NPs with $6.4-\mathrm{J} / \mathrm{cm}^{2}$ fluence, and the raw material of pellets, respectively. The peaks were identified based on the standard data of the Powder Diffraction File of cubic $\mathrm{Gd}_{2} \mathrm{O}_{3}$ (\# 00-0120797) and monoclinic $\mathrm{Gd}_{2} \mathrm{O}_{3}$ (\# 00-043-1015).

Both the peaks of the NPs and raw materials appeared at the same degree, which indicate that miniaturization without any crystal structural change occurred.

No peaks other than $\mathrm{Gd}_{2} \mathrm{O}_{3}$ were observed in both cases, which indicate that no by-products were generated; meanwhile, the NPs were fabricated from raw material by LAL.

\subsection{Primary particle size}

Figure 2 shows the results of the primary particle size observation by SEM. Laser irradiation parameters have a significant effect on morphology [2]. Each grain size is called the primary particle size.

Fig. 2(a) shows the SEM image of the raw material. The raw material's primary particle size was $\sim 200-300 \mathrm{~nm}$. Besides, each grain is condensed because of sintering. Figs. 2(b)-(g) shows the SEM images of the NPs after laser irradiation. Figs. 2(b) and (c) obtained by laser of the low fluence $\left(\sim 2-5-\mathrm{J} / \mathrm{cm}^{2}\right)$ show $200-300$-nm sized primary particles, referred to as 200-300-nm sized coarse NPs herein. Figs. 2(d) and (f) obtained by laser of the middle fluence $\left(\sim 6-\mathrm{J} / \mathrm{cm}^{2}\right)$ show both coarse NPs and NPs below $100 \mathrm{~nm}$. Herein, NPs below $100 \mathrm{~nm}$ were referred to as fine NPs. Figs. 2(e) and (g) obtained by laser of the high fluence ( 9$\mathrm{J} / \mathrm{cm}^{2}$ ) show increased fine NPs.

The primary size difference between the raw material and NPs was considered after laser irradiation. The coarse NPs' primary particle size is $<<$ that of the raw material. It is considered that the coarse NPs were caused by fragmentation that occurred due to the laser's high energy. When the laser hits the bulk, a small part of the bulk material is carved out due to the laser's impact. Thus, coarse NPs emerge. However, the fine NPs' primary sizes are $<$ those of the raw material. It is considered that the fine NPs occurred due to LAL rather than through fragmentation. Since high energy required in LAL [1], when the laser fluence increased at specific points, LAL occur. After the laser hits the bulk, the bulk absorbs the pulse laser. At this time, a rapid transfer of the energy from photons to the lattice occurred that caused significant increase of material temperature. When the temperature exceeds the vaporization temperature, the material evaporates into the plasma plume including energetic species such as atoms, molecules, ions, clusters, and dopants $[1,10]$. As a result of continuing the absorption by bulk, the plasma plume expands surrounding liquid with ultrasound under high pressure. When the kinetic energy of energetic species in the plasma plume decreases due to collision with water molecules [10], inside the plasma plume, atomic nucleation and growth occur. Simultaneously, cavitation bubbles occur around growing NPs. After further ultrasound expansion, cavitation bulb collapsed, and NPs are released to the liquid [36-37]. Thus, when laser ablation occurs, atomic nucleation and growth occur; therefore, smaller primary sizes of the NPs emerge after LAL.
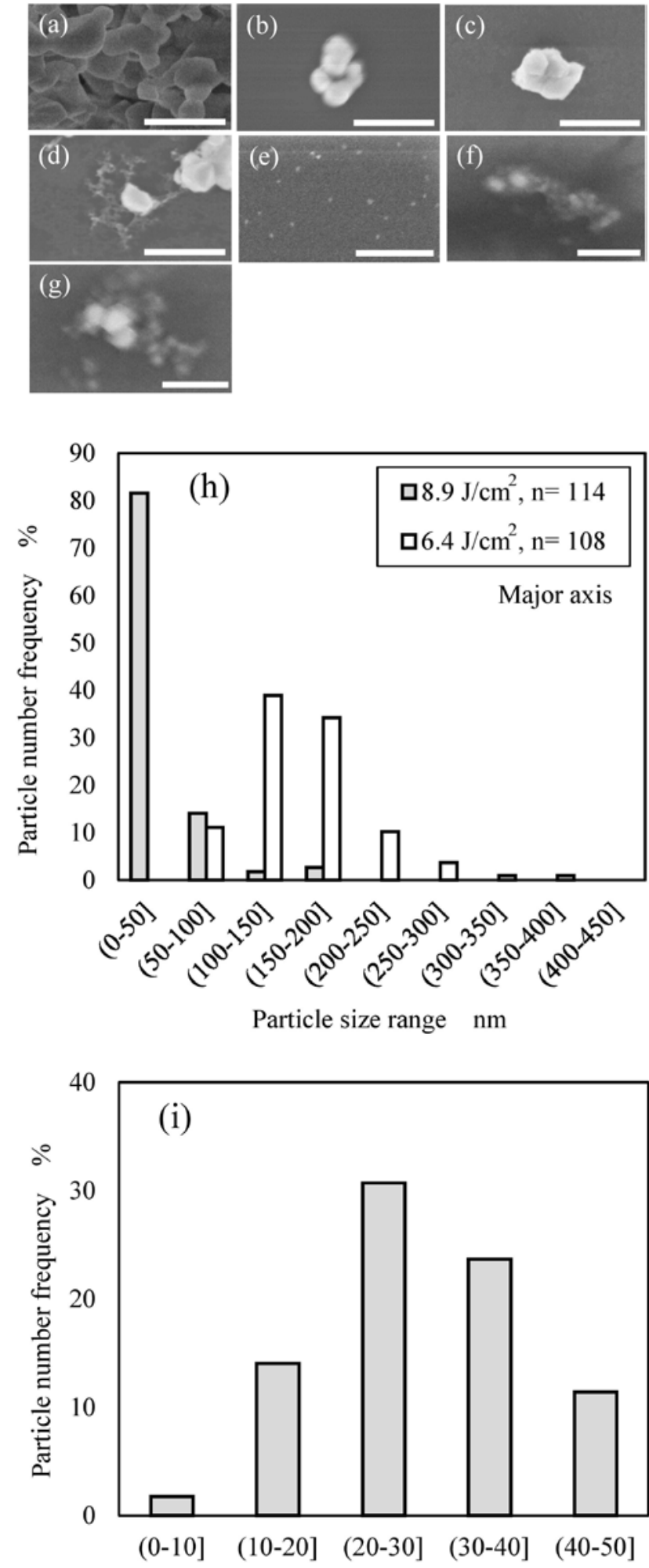

Particle size range $\mathrm{nm}$

Fig. 2 SEM image of (a) raw material, (b) $2.4 \mathrm{~J} / \mathrm{cm}^{2}$, (c) 4.7 $\mathrm{J} / \mathrm{cm}^{2}$, (d) $6.4 \mathrm{~J} / \mathrm{cm}^{2}$, (e) $8.9 \mathrm{~J} / \mathrm{cm}^{2}$, (f) fine NPs at $6.4 \mathrm{~J} / \mathrm{cm}^{2}$, and (g) fine NPs at $8.9 \mathrm{~J} / \mathrm{cm}^{2}$. Scale bar is $500 \mathrm{~nm}$ for (a)-(e) and $100 \mathrm{~nm}$ for (f)-(g). Primary size histogram of (h) NPs at middle and high fluence and (i) NPs at a high fluence in the range of $0-50 \mathrm{~nm}$. 
Similarly, Nunokawa et al. reported $\mathrm{Y}_{2} \mathrm{O}_{3}$ : Er, Yb coarse and fine dot-like NPs after LAL. The diameter of the fine NPs were of the order of $10^{1} \mathrm{~nm}$ and observed with increasing laser density [38].

Figs. 2(h) and (i) show the primary size histograms of the middle and high fluence. Fig. 2(h) shows that at the middle fluence, $100-200$-nm sized coarse NPs were $~ 73 \%$, and fine NPs were $\sim 11 \%$. In contrast, at a high fluence, fine NPs increased to $95.6 \%$. Considering the elimination of NPs of size $\leq 10-\mathrm{nm}$ by the kidney while circulating the body before PDT treatment, fine NPs of size $>10$-nm are preferred. Fig. 2(i) shows the details of the 10-50-nm sized NPs due to the high fluence. The observed particle existence was in the order of 20-30 nm (31\%), 30-40 nm (24\%), $10-20 \mathrm{~nm}$ (14\%), 40-50 nm (11\%), and 0-10 nm (1.8\%). Therefore, $1.8 \%$ of the total NPs of the high fluence are considered to be eliminated; however, $98.2 \%$ of $10-200$ $\mathrm{nm}$ sized NPs can be used for PDT.

\subsection{Secondary particle size}

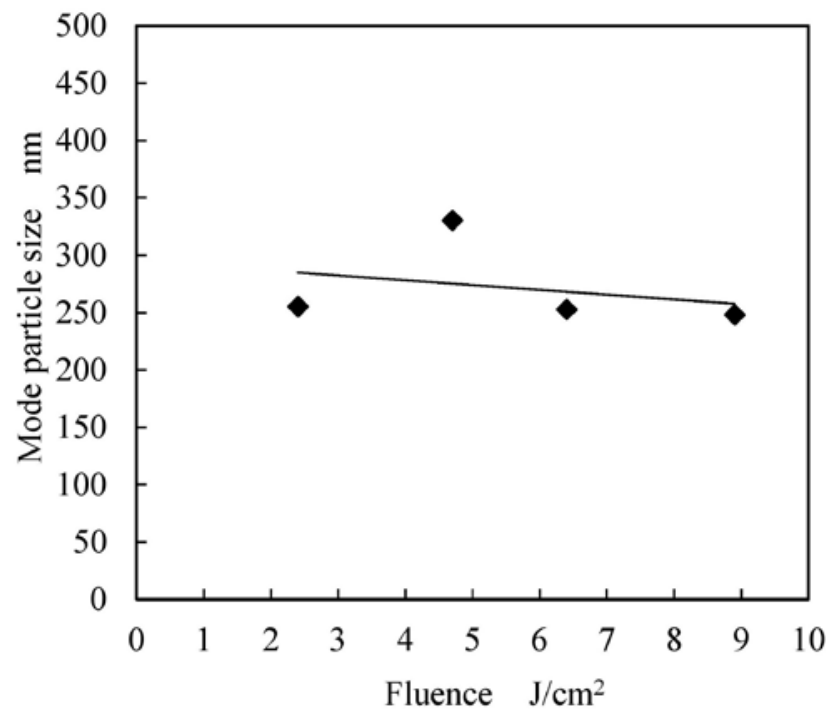

Fig. 3 Secondary particle size of prepared NPs.

Unimodal particle distribution was observed in each fluence from the DLS measurement. The mode particle size was determined and used for comparison in Fig. 3. The entire size of aggregated NPs is called the secondary particle size. As the fluence increased, the secondary particle size did not increase but remained within 200-300 nm. Note that at high fluence, the secondary particle was 248 $\mathrm{nm}$ in size. Despite the increment in fine NPs of $\leq 100 \mathrm{~nm}$, the secondary particle size remained constant at $248 \mathrm{~nm}$. It is considered that the secondary particle increased in size due to aggregation.

Aggregation can be caused by two factors, such as the increased collision frequency and decreased surface potential. The increased collision frequency occurs because the amount of NPs with the laser fluence increases. Thus, the collision frequency increases as the amount of NPs increases, resulting in aggregation. Regarding the decreased surface potential, initially, NPs are highly adhesive and cohesive. Thus, if the particle size reduces to $1-100 \mathrm{~nm}$, chemical and physical properties, and functions change signifi- cantly [39]. Kamiya and Iijima reported their simulation results based on the DLVO theory. They reported that 300nm sized particles could disperse, whereas 20-nm sized particles can aggregate. To disperse $20-\mathrm{nm}$ sized particles, surface potential control is effective. Surface potential can be increased by $\mathrm{pH}$ adjustment and surface coating. Furthermore, they reported that surface coated 20-nm sized particles can be dispersed even in high particle concentration (small distance between the particles) [28]. Fig. 2(i) indicates that $\sim 20-\mathrm{nm}$ sized fine primary NPs mostly exist. Therefore, aggregation was more likely to occur, resulting in secondary particle size of $248 \mathrm{~nm}$ (Fig. 3).

For PDT, 10-200-nm sized particles are expected; thus, agglomeration prevention is essential. Considering the use in the human body, a high biocompatibility surfactant is preferred over $\mathrm{pH}$ adjustment. It is required to be a surfactant that is not recognized as a foreign substance after being taken into a living body. Specific examples of the surfactant having high biocompatibility include polyethylene glycol (PEG), $\mathrm{SiO}_{2}$, and polyvinylpyrrolidone (PVP). PEG is a water-soluble polymer, and is characterized by improved blood retention, low cost, and easy control of molecular weight [40]. Coating the surface of NPs with silicates, which are abundant in the human body, has been shown to enable uncomplicated surface chemistry (such as inhibition of covalent bonds) [41]. PVP is an amphipathic polymer that can be adsorbed on a variety of nanoparticle materials such as gold, silver, iron, iron oxide and alumina [42].

\subsection{Photoluminescence}

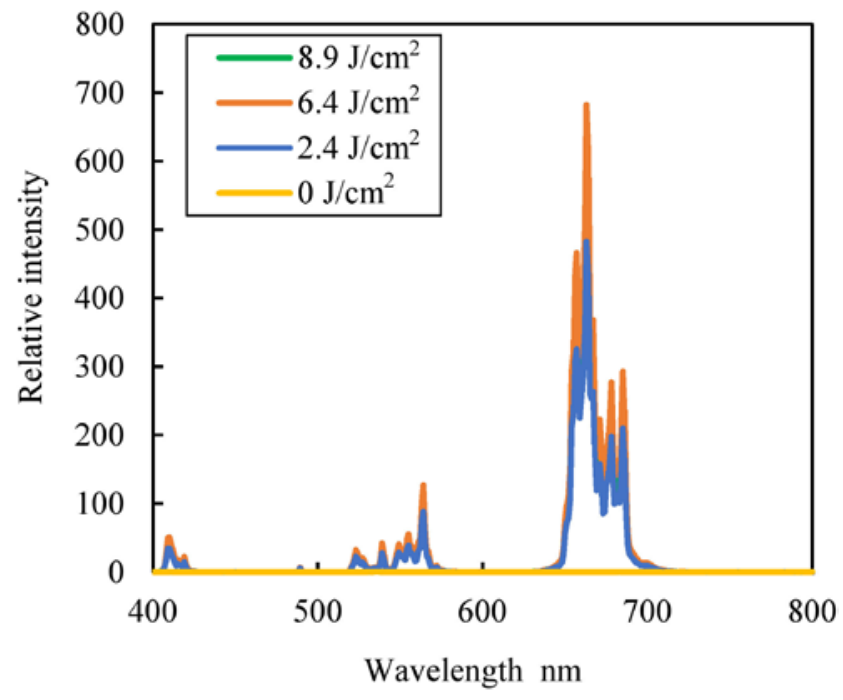

Fig. 4 Photoluminescence spectroscopy of prepared NPs.

As shown in Fig. 4, from the PL measurement, peaks were obtained around 410 (blue), 560 (green), and $660 \mathrm{~nm}$ (red). Visible light was obtained successfully from upconversion NPs irradiating near-infrared light. Only the peak PL intensities in the green and red areas are extracted in Fig. 5. The peak PL intensity increased until the middle fluence as the fluence increased. At a high fluence, the peak PL intensity slightly decreased. It is considered that an increase in the total number of the generated NPs with the fluence increased the peak PL intensity. Also, it is consid- 
ered that a decrease in the peak PL intensity is caused by precipitation due to aggregation and the effect of fine NPs.

The possibility of settling down after aggregation exists, which decreases the floated NPs and weakens the observed PL intensity.

At a high fluence, the amount of fine NPs increased. Since fine NPs have smaller diameters, their specific surface area becomes larger than that of coarse NPs. On the surface, surface defects (kinks and steps), or dangling bonds which have the uncombined hands of the atom exist. These factors have high energy states. If the NPs are large, they are negligible. However, if the NPs become small, they became non-negligible. These surface defects and dangling bond exist in large amounts due to the increased surface area. Therefore, the excitation energy of NPs is consumed by surface defects or dangling bonds. This phenomenon is called nonradiative deactivation, which is turned to heat energy afterward. Thus, part of the excitation energy was consumed by nonradiative deactivation, which decreased the peak PL intensity.

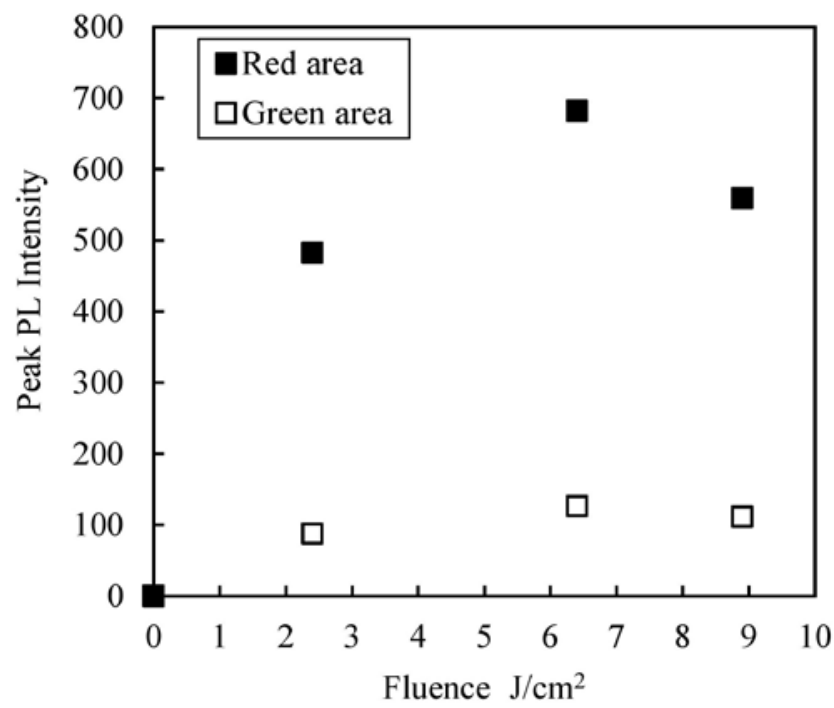

Fig. 5 Peak PL intensity of prepared NPs.

\subsection{Photon number}

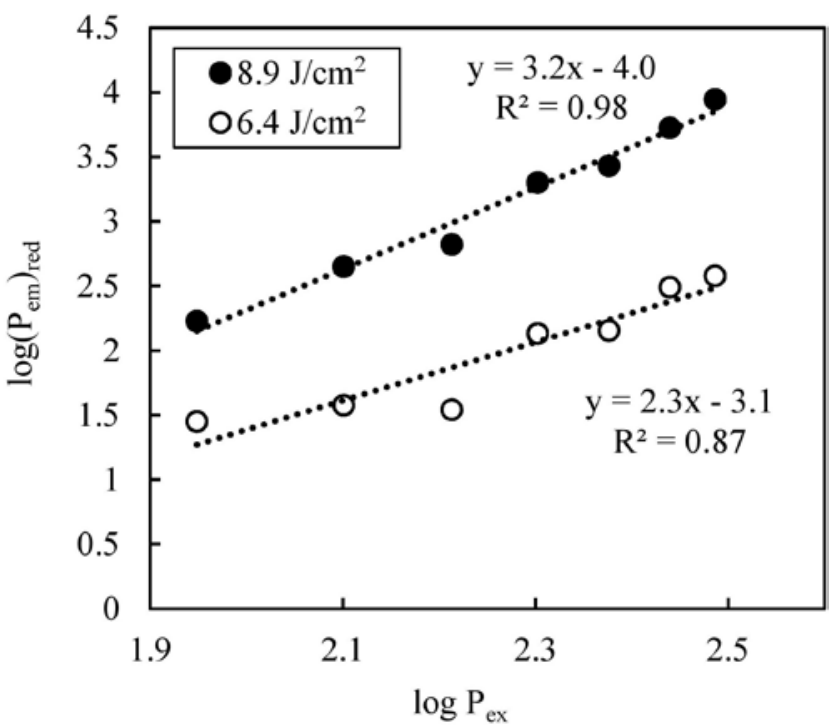

Fig. 6 Photon number of prepared NPs at middle and high fluence.
The relationship between the emission and excitation [43-44] is given below as follows:

$$
P_{\mathrm{em}}=\mathrm{A} P_{\mathrm{ex}}{ }^{n}
$$

where $P_{\mathrm{em}}$ is the emission intensity, $P_{\mathrm{ex}}$ is the excitation intensity, $n$ is the photon number, and A is a constant. Taking the logarithm of both sides gives a linear equation as follows:

$$
\log \left(P_{\mathrm{em}}\right)=n \log \left(P_{\mathrm{ex}}\right)+\mathrm{C}
$$

where $n$ is the slope. Figure 6 shows the measured photon number of the NPs at the middle and high fluence. For the measurement results in the red area (610-750 nm), $n=2.4$ and 3.2 at the middle and high fluence, respectively. Also, $n$ increased toward the high fluence, which can also be attributed to the effect of the fine NPs as mentioned above. Due to the energy loss by nonradiative deactivation, more photon was needed to achieve the excited state.

Thus, to prevent nonradiative deactivation, surface coating by surfactant is effective.

\section{Conclusion}

Here, we fabricated $\mathrm{Gd}_{2} \mathrm{O}_{3}$ :Er,Yb NPs by LAL for biomedical application. Coarse NPs were observed at low fluence, fine NPs emerged at the middle fluence, and fine NPs increased at the high fluence. The secondary particle size was insignificantly changed at each fluence due to aggregation possibility. From the PL measurement results, visible light was successfully upconverted from near-infrared light. Besides, its peak PL intensity increased until the middle fluence and slightly decreased at the high fluence. This trend can be attributed to precipitation due to aggregation and energy loss of nonradiative deactivation caused by fine NPs. Furthermore, the photon number increased toward the high fluence, which can be attributed to the effects of NPs and nonradiative deactivation. Thus, for future research, surface coating by surfactant will be required to prevent aggregation and nonradiative deactivation, increase the PL intensity and decrease the photon number.

\section{Acknowledgments}

The authors thank K. Suda (XRD measurement) at the materials analysis division, open facility center, Tokyo Tech. This study was supported by the Collaborative Research Project of Materials \& Structures Laboratory, Tokyo Tech. The authors would like to thank Enago (www.enago.jp ) for the English language review.

\section{References}

[1] C. Lianwei and H. Minghui: "Laser Surface Structuring of Semiconductors and Functionalization" in Handbook of Laser Micro- and Nano-Engineering ed. by K. Sugioka, (Springer Nature, 2020) p.1.

[2] N. Livakas, E. Skoulas, and E. Stratakis: Optoelectron. Adv., 3, (2020) 190035-1.

[3] S. Dittrich, S. Barcikowski, and B. Gokce: Optoelectron. Adv., 4, (2021) 200072-1.

[4] J. Neddersen, G. Chumanov, and T. M. Cotton: Appl. Spectrosc., 47, (1993) 1959.

[5] F. Mafune, J. Kohno, Y. Takeda, T. Kondow, and H. Sawabe: J. Phys. Chem. B, 104, (2000) 9111.

[6] F. Mafune, J. Kohno, Y. Takeda, T. Kondow, and H. Sawabe: J. Phys. Chem. B, 105, (2001) 5114. 
[7] C. Liang, Y. Shimizu, T. Sasaki, and N. Koshizaki: J. Mater. Res., 19, (2004) 1551.

[8] K. A. Elsayed, H. Imam, M. A. Ahmed, and R. Ramadan: Opt. Laser Technol., 45, (2013) 495.

[9] H. Wang, O. Odawara, and H. Wada: J. Alloys Compd., 683, (2016) 1.

[10] L. Chen, X. Jiang, Z. Guo, Hai Zhu, T. Kao, Q. Xu, G. Ho, and M. Hong: J. Nanomater., 2014, (2014) 1.

[11] K. Soga and M. Kamimura: Drug Deliv. Syst., 33, (2018) 227.

[12] K. Soga and M. Kamimura: Biophys., 57, (2017) 81.

[13] F. Auzel: Chem. Rev., 104, (2004) 139.

[14] F. Auzel: C. R. Acad. Sci. (Paris), 262, (1966) 1016.

[15] F. Auzel: C. R. Acad. Sci. (Paris), 263, (1966) 819.

[16] M. Wang, C. Mi, Y. Zhang, J. Liu, F. Li, C. Mao, and S. Xu: J. Phys. Chem., 113, (2009) 19021.

[17] Q. Zhan, J. Qian, H. Liang, G. Somesfalean, D. Wang, S. He, Z. Zhang, and S. Andersson-Engels: ACS Nano, 5, (2011) 3744.

[18] S. Fischera, A. Ivaturic, P. Jakob, K. W. Krämer, R. Martin-Rodriguez, A. Meijerink, B. Richards, and J. C. Goldschmidt: Opt. Mater., 84, (2018), 389.

[19] Y. Shang, S. Hao, C. Yang, and G. Chen: Nano Mater., 5, (2015), 1782.

[20] W. Yang, X. Li, D. Chi, H. Zhang, and X. Liu: Nanotechnol., 25, (2014), 482001.

[21] D. E. J. G. J. Dolmans, D. Fukumura, and R. K. Jain: Nat. Rev. Cancer, 3, (2003), 380.

[22] C. Wang, L. Cheng, and Z. Liu: Theranostics, 3, (2013), 317.

[23] G. M. F. Calixto, J. Bernegossi, L. M. Freitas, C. R. Fontana, and M. Chorilli: Mol., 21, (2016) 21.

[24] S. Mallidi, G. P. Luke, and S. Emelianov: Trends Biotechnol., 29, (2011) 213.

[25] K. Soga: YAKUGA. ZASSHI, 133, (2013) 355.

[26] T. Sato: Med. Photonics, 7, (2011) 53.

[27] P. Liu, F. Wang, and B. Yang: Solid State Sci., 102, (2020) 106165.

[28] H. Guo, Y. Li, D. Wang, W. Zhang, M. Yin, L. Lou, and S. Xi: J. Alloys Compd., 376, (2004) 23.

[29] H. Li, S. Song, W. Wang, and K. Chen: R. Soc. Chem., 44, (2015) 16081.

[30] A. S. Oliveira, M. T. Araujo, A. S. Gouveia-Neto, J. A. M. Neto, A. S. B. Sombra, and Y. Messaddeq: Appl. Phys. Lett., 72, (1998) 753.

[31] X. Tian, F. Yang, C. Yang, Y Peng, D. Chen, J. Zhu, F. He, L. Li, and X. Chen: Int. J. Nanomed., 9, (2014) 4043.

[32] T. Ikehata, Y. Onodera, T. Nunokawa, T. Hirano, S. Ogura, T. Kamachi, O. Odawara, and H. Wada: Appl. Surf. Sci., 348, (2015) 54.

[33] Y. Matsumura and H. Maeda: Cancer. Res., 46, (1986) 6387.

[34] S. M. A. Sadat, S. T. Jahan, and A. Haddadi: J. Biomater. Nanobiotechnol., 7, (2016) 91.

[35] M. Yokoyama: Drug Deliv. Syst., 33, (2018) 89.

[36] J. Liu, L. Huang, X. Tian, X. Chen, Y. Shao, F. Xie, Dihu Chen, and L. Li: Int. J. Nanomed., 12, (2017) 1.

[37] S. Hashimoto: Laser Res., 45, (2017) 1.

[38] T. Nunokawa, Y. Onodera, M. Hara, Y. Kitamoto, O. Odawara, and H. Wada: Appl. Surf. Sci., 261, (2012) 118.
[39] H. Kamiya and M. Iijima: The Micromeritics, 55, (2012) 12.

[40] I. Sugiyama and Y. Sadzuka: Drug Delivery Syst., 31, (2016) 275.

[41] K. Fujii, Y. Kitamoto, M. Hara, O. Odawara, and H. Wada: J. Lumin., 156, (2014) 8.

[42] R. Riedel, N. Mahr, C. Yao, A. Wu, F. Yang, and N. Hampp: Nanoscale, 12, (2020) 3007.

[43] Y. Onodera, T. Nunokawa, O. Odawara, and H. Wada: J. Lumin., 137, (2013) 220.

[44] M. Pollnau, D. R. Gamelin, S. R. Luthi, and H. U. Gudel: Phys. Rev. B, 61, (2000) 3337.

(Received: June 11, 2021, Accepted: October 9, 2021) 